\title{
Genome rearrangements in bacterial genomes
}

\author{
O.O. Bochkareva ${ }^{1 *}$, M.S. Gelfand ${ }^{1,2,3,4}$ \\ ${ }^{1}$ Institute of Information Transmission Problem RAS, Moscow, Russia \\ ${ }^{2}$ Lomonosov State University, Moscow, Russia \\ ${ }^{3}$ Higher School of Economics, Moscow, Russia \\ ${ }^{4}$ Skolkovo Institute of Science and Technology, Moscow, Russia \\ *e-mail: olga.bochkaryova@gmail.com
}

Key words: bacterial evolution, genome rearrangements, phylogeny reconstruction

Motivation: Gene order in prokaryotes is relatively poorly conserved making it a convenient tool for the analysis of the species and strain evolution, when changes in protein, and even gene sequences do not provide sufficient resolution [1]. In addition, genome rearrangements are less sensitive to homologous recombination and hence allow for an alternative approach to construction of phylogenetic trees, as even a small number of genome rearrangements may resolve topological ambiguities in a phylogenetic tree [2]. Results: For several bacterial species we reconstructed phylogenetic trees based on sequences similarities and gene order. Our results show that rearrangement rates differ dramatically in different bacterial species, that is likely to be related to the adaptation to different ecological niches. For newly formed pathogens such as Y. pestis and B. mallei with a particularly high rate of rearrangements we revealed the correlation between mutations rates and inversions rates. Analysis of contradictions between the obtained evolutionary trees yielded numerous parallel rearrangements. Numerous gene losses likely have been caused by a high rate of intragenomic recombination between limited number of repeated elements such as transposases and 16S-23S rRNA clusters, that also creates the plasticity of gene order in chromosomes. We revealed cases when inversions found in separated branches in the phylogenetic trees may result in phase variation. Also analysis of syntenies positions allowed us to reveal gene cassettes that are spreading horizontally such as iron uptake genes cluster in B. cepacia group.

Conclusion: Our data indicate that an integrated analysis of sequence-based and inversion-based trees enhances the resolution of phylogenetic reconstruction. At that, inversions may resolve branches with low bootstrap support; on the other hand, sequence analysis may distinguish between parallel inversions and single inversion propagated by homologous recombination. The reconstructed patterns of rearrangements indicate strong avoidance of large inter-replichore inversions and translocations that is likely to be caused by selection against transfer of large fractions of genes between the leading and the lagging strands.

Acknowledgements: Supported by the Russian Science Foundation under grant 14-5000150.

\section{References}

1. Wolf Y.I., Rogozin I.B., Kondrashov A.S., Koonin E.V. (2001). Genome alignment, evolution of prokaryotic genome organization, and prediction of gene function using genomic context. Genome Res. 11(3):356-72.

2. Darling A.E., Miklós I., Ragan M.A. (2008). Dynamics of genome rearrangement in bacterial populations. PLoS Genet. 4:e1000128. 